\title{
Impact of Infographics and Visual Representation on Statistical Enquiry
}

\author{
Saqib Ahmad (Master in Commerce) \\ House No. 80/4-D, Street No. 35, F-6/1, Islamabad
}

\begin{abstract}
The concept of statistical enquiry is mostly related to gathering of data, search of knowledge by using some conventional methods. Today, it become easy for us to get exact information we needed with the help of modern tools, techniques and methods. Still, we are far from gathering the authentic information related to problem and research purpose. This paper will cover up the entire scenario that most of the countries should adopt the new easy and convenient way of finding true information related to statistics.
\end{abstract}

DOI: $10.7176 / \mathrm{JESD} / 11-5-01$

Publication date:March $31^{\text {st }} 2020$

\section{Introduction}

There are many underdeveloped countries that are using the same old paper work of collecting the statistical information. It has been seen that most of the census conducted in these countries are based entirely on single paper book. How can we say that the information is collected is entirely true? Or how can we judge the information collected is hundred per cent accurate?

We all know that when we talk about underdeveloped countries the challenge of collecting information is the toughest part that is because of most of the families are uneducated, this can only bring wrong information. If we want to know how people want to see the change in particular area, will they able to easily describe us or is there any way they can tell us exact true information rather than telling in simple plain words? Here, visual representation and infographics plays an important role.

Let me make it simple for you and before moving any further it's important that you should know what these two heads are:

\section{Infographics \& Visual Representation}

"Info" as a name suggests taken from information and "graphics" are anything that are visually live. So, we can say any information that can be seen as a graphical object or even a picture is visual representation of a data. Now, please consider four main points below to help you understand better about the infographics and visual representation.

- We know that statistics do possess graphs, charts and pictogram, we are talking about advance graphical representation and steps of gathering data and research.

- We will cover $80 / 20$ rules, $80 \%$ graphics do tells best about the issue rather than a paper full of text, It will take less space, less time and more accurate result.

- We do requires the help of designers in this process, As simple charts diagrams can be made by anyone, For best result and attractive graphic representation we need expert in the field of graphic designing.

- Visual representation does not involve only in pictures or diagram rather this also covers the video applications and voice over techniques for using online platforms to provide statistical information's.

\section{Comparison:}

Let's take a simple example and see how it works, for example we finds out the following facts while researching the group of students in class. Let's compare with simple text and with graphical representation.

\section{Plain Text:}

We find out that every class has 8 students and out of these 5 are boys and 3 are girls. We selected 4 students from each class and our research find out that 1 of the girls and 3 of the boys in each class have aqua eyes. 


\section{Graphical Representation:}
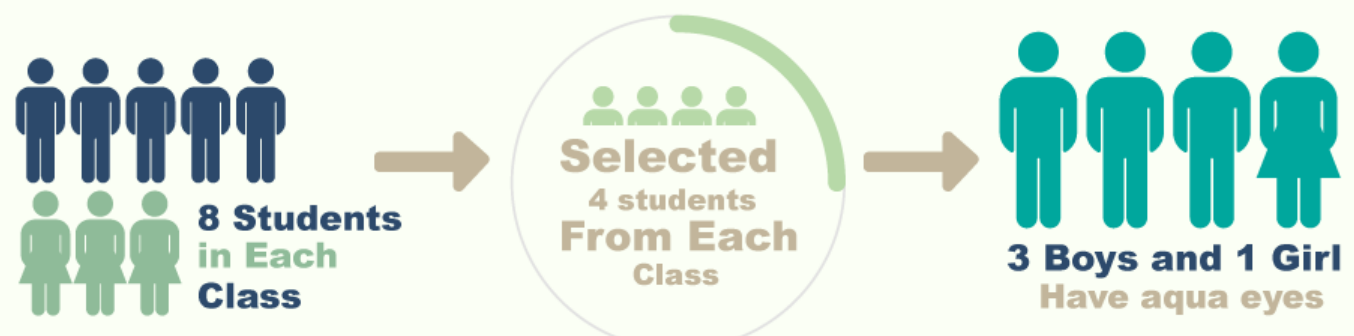

See, how much difference this creates, visual made it look more attractive and easy to understand at same time. Another thing is to focus that you will remember most of the scenario in graphic form rather than in plain text.

\section{Subtle art of Data Collection, Chart}

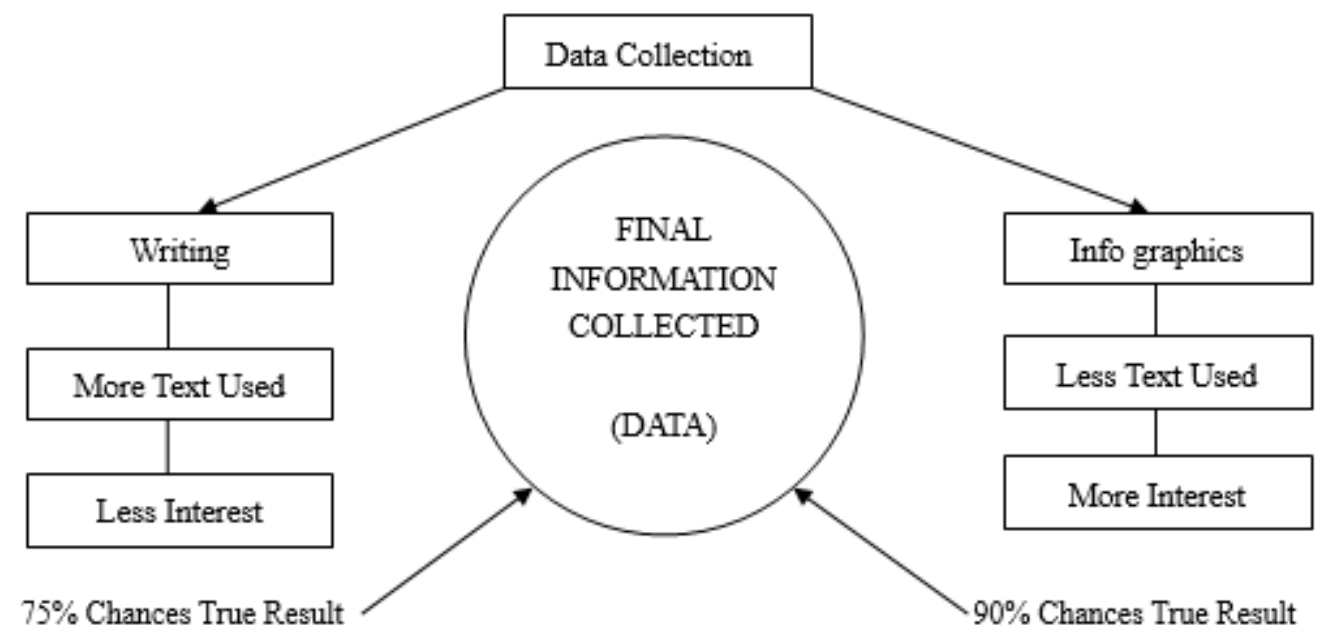

\section{Subtle art of Data Collection, Chart}

\section{Detail of Chart:}

While collecting data for any kind of research we do lots of data gathering. The question arises which method we use to gather the accurate information as much as possible. In above chart you can see that plain text do gather data that can be in any form like questioner and choosing right option or direct talk with the target, however, if we use the technique and convert these into graphical form to gather the data then it will make data gathering more interesting and attractive at same time.

Again here, 80/20 rule will work best. More picture and diagram is always better as compared to using the text that does bring information but interest will be less as compared to graphical form of collecting of data. We will get more accurate information in graphical form but not $100 \%$ accurate as statistics is not always cent per cent true.

In above diagram both situations brings results but a little change can make a great difference in data collection and solving problem can become lot easier as compared to data we gathered in form of plain text.

\section{Types of Infographics and Visual Representations we can use:}

Now, when we talk about visual representation then there are literally tons of ideas you can implement. Below in my opinion are some of the best ways to gather data. Let's divide on the basis of use and complexity of methods.

\section{1- Basic Tools and Techniques:}

These tools can be used by anyone and are less expensive and easy to use, let's see each one of them

\section{Websites:}

As always one of the best ways to implement infographics is to use the website which is really easy and same time you will get information. You can send emails to your subscribers list, ask them to fill out the simple survey based on graphics and this way you can get lots of true information. 
Printed Materials:

Infographics are not limited to internet world and I never said that, however, there are other convenient ways of gathering data and one of them is by printing graphics on any type of material. You can use papers, banners and any type of items that can help you collecting information.

\section{Video Making:}

Advertisement plays an important role both in gathering and showing information but, how they can help us gather the information? It's simple, use on point screening of material and in return asks general questions from the audience.

\section{2- Advance Tools and Techniques:}

These tools requires some extra effort as may involve in some expenses but still, can be easily taken for data collection. Let's see each one of them

\section{Animation Techniques:}

People love to see animation as they capture human interest more. You can use the minimal concepts most of the time as these are simple and cost effective. Animation however, cost more as compared to using the simple tool but result can be much better.

\section{Live Classes and Webinar:}

The only demerit of using this method is the gathering of audience, you can gather the data from them but how you will bring the right audience for your data collection process. This method usually involves trusting someone so, it's hard to start but less cost effective.

\section{Scenario Based Activity:}

One of the most interesting and latest techniques used by most of the companies is scenario based activity. It's like storyboard where you have to select a character which is graphical based and play the game to enter the information. Currently this method is used by many employers to hire the perfect candidate for the job which is actually working very well.

\section{Conclusion}

As we can see how much a small change in data collection can bring a great result. There are lots of hurdles in way of collecting the data and by using this method we can easily collect the information that is needed to justify the research problem. You might need to invest further on collecting data like making info graphics do cost you a little but the end result is much better as compared to collecting data using the plain text. In fast phase world activities we need to change the way we collect data. Collection is never hard if we use right methods but the information we need is accurate, so, the problem solution can be easy.

Further there is possibility that this method will be adopted in developed countries soon as already we can see a great change in blogs and online websites, They tend to show more infographics to their website in order to help people understand better information they are providing and yes! It is helping them in number of ways like traffic increase, better conversion and more customer retention. So, why not using this method collecting data in real world? It's simple and convenient.

\section{References}

Allyn and Bacon (1997), "Infographics a journalist's Guide”, James Glen Stovall, University of Alabama. W.H. Freeman (1991), "Statistics: concepts and controversies"

Wadsworth \& Brooks/Cole "Statistics: a guide to the unknown 1989"

Krieger 1992 "Statistical graphics: design principles and practices"

"Statgraphics: statistical graphics system" 1991, STSC Edition 5 